\title{
Preoperative Laboratory Testing by Surgeons: Implication on Anaesthetic Management
}

\author{
Akwasi Antwi-Kusi1 ${ }^{*}$, William Addison1, Bright Ighodaro Obasuyi², Philip Domoyeri ${ }^{3}$ \\ ${ }^{1}$ Department of Anaesthesia and Intensive Care, Komfo Anokye Teaching Hospital, Kumasi, Ghana \\ ${ }^{2}$ Department of Anaesthesiology, University of Port Harcourt Teaching Hospital, Port Harcourt, Nigeria \\ ${ }^{3}$ Koforidua Regional Hospital, Koforidua, Ghana \\ Email: *antwikusi@yahoo.com,waddison@knust.edu.gh,pffmph@yahoo.com,domoyeri@yahoo.com
}

How to cite this paper: Antwi-Kusi, A., Addison, W., Obasuyi, B.I. and Domoyeri, P. (2019) Preoperative Laboratory Testing by Surgeons: Implication on Anaesthetic Management. International Journal of Clinical Medicine, 10, 345-352.

https://doi.org/10.4236/ijcm.2019.106027

Received: May 3, 2019

Accepted: June 14, 2019

Published: June 17, 2019

Copyright (c) 2019 by author(s) and Scientific Research Publishing Inc. This work is licensed under the Creative Commons Attribution International License (CC BY 4.0).

http://creativecommons.org/licenses/by/4.0/

\begin{abstract}
Background: Request for preoperative laboratory investigations is usually done by surgeons. On some occasions, the patient may come with laboratory investigations that have been requested by the primary physician. This occurs in situations where the primary physician saw the patient first and referred to the surgeon. There is usually no indications based on history or physical examination before these laboratory request is done but rather on speculations that the "anaesthetist may require them". This is done in order to avoid cancellation or delay of cases. The aim of this study was to find out how tests ordered in Komfo Anokye Teaching Hospital (K.A.T.H.) by the surgeons affected the decisions of the anaesthetists in the perioperative management of the patients. Methodology: This was a prospective, cross sectional study of patients undergoing elective surgery at K.A.T.H from $1^{\text {st }}$ to $31^{\text {st }}$ March 2014. A quantitative technique was used to effectively quantify laboratory results that were contained in a patient's folder before an elective surgical procedure. A close and open-ended questionnaire was developed and answered by reviewing patient's folders during the pre-anesthesia assessment. Data were analyzed using Statistical Package of Social Sciences (SSPS) version 22. Results: The average age of patients studied was 50 years. Abnormal test results did not influence the anaesthetic management in $70.5 \%$ of cases but led to either delay or cancelation of cases or requirement for transfusion of blood or blood products in $29.5 \%$ of cases. Conclusion: Preoperative laboratory tests ordered by Surgeons in KomfoAnokye Teaching Hospital do not significantly influence the anaesthetic managements of patients.
\end{abstract}

\section{Keywords}

Preoperative, Laboratory Investigations, Surgeons, Anaesthetic Management, Implication 


\section{Introduction}

A preoperative laboratory test is done as part of the requirement for pre-anesthetic assessment. Traditionally a lot of importance is attached to these laboratory tests because of its importance in the evaluation of the patient's fitness for anaesthesia and surgery [1] [2].

Even though these laboratory tests are important, it should not be requested arbitrary. There should always be an indication for requesting any laboratory investigation. The request should be based on the patient's history, physical examination and the risk of the surgical procedure. A lot of institutions have their guidelines for ordering preoperative laboratory tests. However, a test is likely to be indicated only if it can correctly identify abnormalities and will change the diagnosis, the management plan, or the patient's outcome [3] [4].

The ordering of preoperative laboratory tests should hinge on specific clinical indications that are likely to increase the peri-operative risk and not simply based on the fact that the patient is undergoing a surgical procedure [5].

The practice of ordering routine laboratory tests for all patients undergoing surgery without taking into consideration, the patient's age or medical history is no longer considered medically appropriate [5].

In a lot of institutions, it is the surgeon or the primary physician who orders the pre-operative laboratory test. In most situations, these tests are ordered not based on any specific indication or the history and examination obtained from the patient. In most situations, the ordering of the laboratory tests is based on the speculation that the anaesthetists may require them to proceed with the surgery and thus avoid delays and cancellations [5] [6].

A study conducted by Vogt A. W et al. concluded that an average of $72.5 \%$ of tests ordered by surgeons was considered not indicated by the anaesthesiologists. Anaesthesiologists are the peri-operative medicine experts and are best qualified to establish an appropriate and necessary preoperative laboratory and diagnostic tests for intra-operative anaesthesia management [5]. Routine laboratory testing before elective surgery in healthy patient has limited clinical relevance and will seldom change the anaesthetic care plan or influence outcome [7]; however, the use of routine laboratory investigations before elective surgery is rampant [2]. This study sought to find out how tests ordered in Komfo Anokye Teaching Hospital by the surgeons affected the decisions of the anaesthetists in the peri-operative management of the patients.

\section{Methods}

This was a prospective, cross-sectional survey which involved patients undergoing elective surgical procedures at the Komfo Anokye Teaching Hospital, Kumasi, Ghana. After ethical clearance from the committee for human research publication and ethics, a census from $1^{\text {st }}-31^{\text {st }}$ March 2014 was done to collect data from folders of patients presenting for various surgical procedures that met the inclusion criteria, and the anaesthetist who anaesthetized the patients filled 
the final part of the questionnaire. The folders of all the patients presenting for elective surgery during the study period were reviewed. A quantitative technique was used to effectively quantify laboratory results that were contained in a patient's folder before an elective surgical procedure. Close and open-ended questionnaire was developed and answered by reviewing patient's folders during the pre-anaesthesia assessment. Part of the questionnaire was answered by the attending anaesthetist immediately after the surgical procedure had been performed. In all, a total of one hundred and sixty five patients were enrolled in the study.

\section{Data Analysis}

The data was analyzed using Statistical Package of Social Sciences SPSS version 22 and Microsoft Excel 2010. The results were presented in the form of frequency tables.

\section{Results}

The study population was 165 candidates with a mean age of 50 years and a male to female ration of 1:1.1. At the time of pre-anaesthetic review, all the candidates had at least one laboratory result. $98.8 \%$ of the laboratory tests were ordered by the surgical team while the anaesthetist was responsible for only $1.2 \%$ of all laboratory requests (Table 1 ).

Table 2 shows that complete blood count was ordered most frequently among candidates studied (98.8\%) while thyroid function test was the least ordered in $5.5 \%$ of the population. Of the 163 candidates in whom $\mathrm{CBC}$ was requested, results were normal in $122(74.8 \%)$ and abnormal in 41 (25.2\%). Abnormal results were also seen in $14.4 \%, 3.2 \%, 20.9 \%$ and $46.2 \%$ of candidates who had renal function test (RFT), serum electrolytes, liver function test (LFT) and coagulation studies done respectively.

These abnormal test results did not however influence the anaesthetic management in $70.5 \%$ of cases but led to either delay or cancelation of cases or requirement for transfusion of blood or blood products in 29.5\% of cases (Figure 1 and Table 3).

Furthermore, Table 4 reveals that most of the tests ordered were not necessary according to the Canadian Anesthesiologist Society guidelines for pre-operative testing (Table 5) and were thus routinely ordered.

\section{Discussions}

Our study shows that laboratory tests ordered by surgeons were not indicated in 39.3\% of cases when compared to the Canadian Anesthesiologist Society. Abnormal results did not influence the anaesthetic management in $70.5 \%$ of cases but rather led to either delay or cancelation or requirement for transfusion of blood or blood products in $29.5 \%$ of cases. Most of the changes were based on very low haemoglobin levels. This trend is similar to the findings of Riyard M et al. 
Table 1. Demographic and pre-surgical characteristics.

\begin{tabular}{ccc}
\hline Characteristics Value & Percentage & \\
\hline Number of patients & 165 & \\
Mean age (years) \pm SD & 50 & 98.8 \\
Male to female ratio & $1: 1: 1$ & 1.2 \\
\hline Who orders Lab Investigations? And frequency of pre-anaesthetic lab results \\
Surgeons & 163 & 100 \\
Anaesthetists & 2 & \\
Pre-anaesthetic lab results & 165 &
\end{tabular}

Table 2. Frequency of tests ordered.

\begin{tabular}{ccccc}
\hline Lab test & Not Requested & Requested & Normal & Abnormal \\
\hline Complete blood count & $2(1.2 \%)$ & $163(98.8 \%)$ & $122(74.8 \%)$ & $41(25.2 \%)$ \\
Renal function test & $40(24.2 \%)$ & $125(75.8 \%)$ & $107(85.6 \%)$ & $18(14.4 \%)$ \\
Bldgrping and matching & $60(36.4 \%)$ & $105(63.6 \%$ & $105(100 \%)$ & $0(0.0 \%)$ \\
Serum electrolytes & $71(43.0 \%)$ & $94(57.0 \%)$ & $91(96.8 \%)$ & $3(3.2 \%)$ \\
Liver function test(LFT) & $117(70.9 \%)$ & $48(29.1 \%)$ & $38(79.1 \%)$ & $10(20.9 \%)$ \\
Urinalysis & $146(88.5 \%)$ & $19(11.5 \%)$ & $19(100 \%)$ & $0(0.0 \%)$ \\
Blood glucose & $147(89.1 \%)$ & $18(10.9 \%)$ & $18(100 \%)$ & $0(0.0 \%)$ \\
Coagulation studies & $152(92.1 \%)$ & $13(7.9 \%)$ & $7(53.8 \%)$ & $6(46.2 \%)$ \\
Thyroid function test & $156(94.5 \%)$ & $9(5.5 \%)$ & $9(100 \%)$ & $0(0.0 \%)$ \\
Haemoglobin & $165(100 \%)$ & $0(0.0 \%)$ & $0(0.0 \%)$ & $0(0.0 \%)$ \\
Haematocrit & $165(100 \%$ & $0(0.0 \%)$ & $0(0.0 \%)$ & $0(0.0 \%)$ \\
Pregnancy evaluation & $165(100 \%)$ & $0(0.0 \%)$ & $0(0.0 \%)$ & $0(0.0 \%)$ \\
\hline
\end{tabular}

Table 3. Changes made in anaesthetic management based on laboratory results.

\begin{tabular}{ccccccc}
\hline & CBC & CS & SE & RFT & LFT & Total \\
\hline $\begin{array}{c}\text { Cancellation/delay } \\
\text { of surgery }\end{array}$ & $3(7.3 \%)$ & $0(0.0 \%)$ & $0(0.0 \%)$ & $0(0.0 \%)$ & $0(0.0 \%)$ & $3(3.9 \%)$ \\
$\begin{array}{c}\text { Transfusion of } \\
\text { Bld/Bld products }\end{array}$ & $12(29.3 \%)$ & $0(0.0 \%)$ & $1(33.3 \%)$ & $4(22.2 \%)$ & $3(30.0 \%)$ & $20(25.6 \%)$ \\
No changes made & $26(63.4 \%)$ & $6(100 \%)$ & $2(66.7 \%)$ & $14(77.8 \%)$ & $7(70.0 \%)$ & $55(70.5 \%)$ \\
Total & $41(100 \%)$ & $6(100 \%)$ & $3(100 \%)$ & $18(100 \%)$ & $10(100 \%)$ & $78(100 \%)$ \\
\hline
\end{tabular}

Bld-blood; CBC—complete blood count; CS—coagulation studies; SE-serum electrolytes; RFT-renal function test; LFT-liver function test.

[8]. In their study, only $30(3.38 \%)$ of 839 patients in whom complete blood count was indicated and $2(0.18 \%)$ of 1091 patients in whom the test was indicated had interventions respectively. No interventions occurred in patients with 
Table 4. Number of investigations that were indicated or not indicated according to the Canadian Anesthesiology Society guidelines for preoperative laboratory testing.

\begin{tabular}{ccc}
\hline Laboratory test & Indicated & Not indicated \\
\hline Complete blood count (\%) & $124 / 163(76.1)$ & $39 / 163(23.9)$ \\
Serum electrolytes (\%) & $56 / 94(59.6)$ & $38 / 94(40.4)$ \\
Renal function test (\%) & $57 / 125(45.6)$ & $68 / 125(54.4)$ \\
Coagulation studies (\%) & $8 / 13(61.5)$ & $5 / 13(38.5)$ \\
Average percentage & 60.7 & 39.3 \\
\hline
\end{tabular}

Table 5. The Canadian Anaesthesiology Society Guidelines for preoperative laboratory testing.

\begin{tabular}{|c|c|}
\hline Test & Indications \\
\hline Complete Blood Count & $\begin{array}{l}\text { - Matient more than } 60 \text { years of age } \\
\text { or "group and match" } \\
\text { - Chronic cardiovascular, pulmonary, renal or } \\
\text { hepatic disease } \\
\text { - Malignancy } \\
\text { - Known or suspected anemia, bleeding } \\
\text { diathesis or myelosuppression } \\
\text { - Anticoagulant therapy }\end{array}$ \\
\hline Coagulation profile & $\begin{array}{l}\text { - Anticoagulant therapy } \\
\text { - Bleeding diathesis, family history of bleeding } \\
\text { disorder } \\
\text { - } \text { History of DVT or pulmonary embolism } \\
\text { - } \quad \text { Liver disease, renal failure } \\
\text { - Malignancy with concurrent } \\
\text { radio-chemotherapy }\end{array}$ \\
\hline Electrolytes and creatinine levels & $\begin{array}{l}\text { - } \text { Age more than } 60 \text { years } \\
\text { - Hypertension } \\
\text { - } \text { Renal disease } \\
\text { - } \text { Diabetes } \\
\text { - } \text { Pituitary or adrenal disease } \\
\text { - } \text { Digoxin or diuretic therapy, or other drug } \\
\text { therapies affecting electrolytes }\end{array}$ \\
\hline
\end{tabular}

abnormal coagulation profile as was the case with our study. Interventions were also low in patients with abnormal serum electrolytes. The lower figures for this study may be explained by the relatively larger population when compared to ours.

In a randomized, single-blind, prospective, controlled pilot study to determine whether indicated preoperative testing can be eliminated without increasing the perioperative incidence of adverse events in selected patients undergoing ambulatory surgery, one thousand and sixty-one eligible patients were randomized either to have indicated preoperative testing or no preoperative testing. In the indicated testing group, patients received indicated preoperative testing: a 


\section{Influence of abnormal results on anaesthetc management}

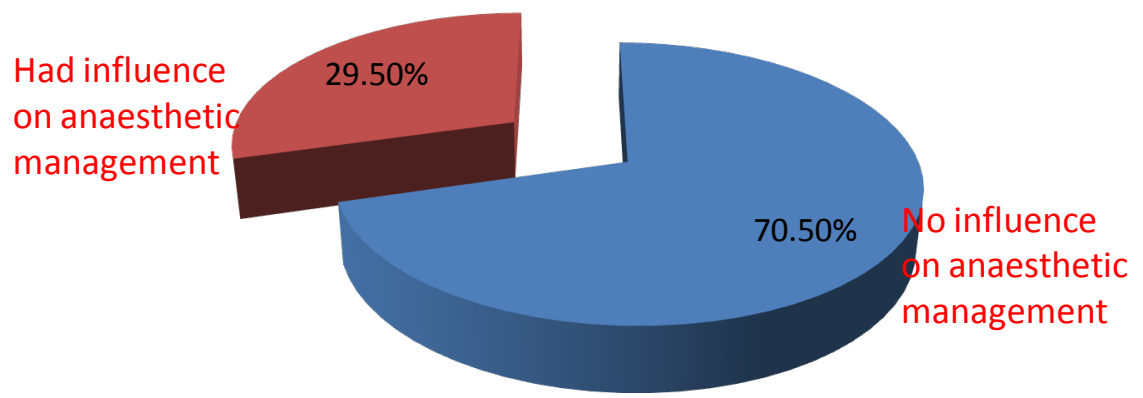

Figure 1. Influence of abnormal results on anaesthetic management.

complete blood count, electrolytes, blood glucose, creatinine, electrocardiogram, and chest radiograph according to the Ontario Preoperative Testing Grid as per current practice, whereas in the no testing group, no testing was ordered. Although patients' age, gender, American Society of Anesthesiologists status, type of surgery, and anesthesia were similar between the two groups, there were no significant differences in the rates of perioperative adverse events and the rates of adverse events within 30 days after surgery between the no testing group and the indicated testing group [9].

In the early 1940s when preoperative assessment evolved, preoperative investigations were based on thorough history and physical examination during preoperative visit. Laboratory test ordered was done selectively to confirm diagnosis [10]. However in the late 1960s when test ordering became easier because of the introduction of biochemical auto-analyzers routine testing became common because it was equated to efficient care [2].

Many hospitals adopted this behaviour to perform a series of laboratory test prior to surgical operations with the assumption that voluminous information would enhance the safety of surgical patients [2]. The practice continued without any scientific evidence until Kaplan et al. published an academic paper to debunk the practice. In their study a retrospective review of charts of over 2000 elective surgical patients who underwent a battery of tests including complete blood cell count, differential blood count, prothrombin time, glucose level, serum electrolytes, creatinine, platelet count, etc, they demonstrated that only 96 (22\%) tests revealed abnormalities. Of 96 abnormal test results, only 10 could not be determined by history and examination, of which only 4 were of actual clinical significance [11].

Although some test abnormalities are clearly of concern (e.g. raised blood sugar), others may have little or no effect on peri-operative anaesthetic management or outcome e.g. white blood cell count [2]. Available consensus indicates that only selective tests should be advised, considering patients health 
status, presence of medical diseases, current medication, invasiveness or risk of proposed operative procedure (minimally, moderately or highly invasive), and potential for blood loss. The tests should be obtained for specific clinical indication (e.g. obtain blood glucose in a known or suspected patient of diabetes or require complete blood count in surgeries where moderate or severe blood loss is expected) that may increase peri-operative risk or influence management of anaesthesia or surgery and not simply because the patient is to undergo surgery [2].

Healthy patients of ASA physical status I and II without co-existing medical condition undergoing minimally invasive outpatient surgery may require no routine investigations, whereas those scheduled for moderately or severely invasive surgery which causes major physiological stress, a few baseline tests may be done. Further testing is needed only as per specific medical condition. In older patients with medical diseases, the likelihood of abnormal tests is higher; therefore more liberal testing may be done. However, using age as a criterion for routine tests is debated and ASA physical status and risk of surgery are considered better predictors of surgical outcome in elderly patients [10].

\section{Conclusion}

Preoperative laboratory tests are ordered by surgeons in Komfo Anokye Teaching Hospital. Most of the results are normal and even the abnormal results do not significantly influence the anaesthetic managements of patients. We recommend that ordering of preoperative investigation should be the responsibility of the anaesthetist during preoperative assessment; the practice of surgeons ordering preoperative laboratory investigations for the anaesthetist should be minimised.

\section{Conflicts of Interest}

The authors declare no conflicts of interest regarding the publication of this paper.

\section{References}

[1] (2008) Standards and Practice Parameters, ASA House of Delegates. Routine Preoperative Laboratory and Diagnostic Screening. Google Search.

[2] Kumar, A. and Srivastava, U. (2011) Role of Routine Laboratory Investigations in Preoperative Evaluation. Journal of Anaesthesiology Clinical Pharmacology, 27, 174-179. https://doi.org/10.4103/0970-9185.81824

[3] Hepner, D.L. (2009) The Role of Testing in the Preoperative Evaluation. Cleveland Clinic Journal of Medicine, 76, S22-S27. https://doi.org/10.3949/ccjm.76.s4.04

[4] Feely, M.A., Collins, C.S., Daniels, P.R., Kebede, E.B., Jatoi, A. and Mauck, K.F. (2013) Preoperative Testing before Noncardiac Surgery: Guidelines and Recommendations. American Family Physician, 87, 414-418.

[5] Fischer S.P., Bader, A.M. and Sweitzer, B. (2009) Preoperative Evaluation. In: Miller, R.D., Eds., Millers Anesthesia, 7th Edition, Chapter 34, Elsevier Churchill Li- 
vingstone, London.

[6] Brown, S.R. and Brown, J. (2011) Why Do Physicians Order Unnecessary Preoperative Tests? A Qualitative Study. Family Medicine, 43, 338-343.

[7] Smetana, G.W. and Macpherson, D.S. (2003) The Case Against Routine Preoperative Laboratory Testing. Medical Clinics of North America, 87, 7-40.

[8] Riyad, M., Al Saied, G., Alshareef, A., Uddin, S., Abdulkarim, Y and Muhaimeed, K. (2013) Usefulness of Routine Preoperative Laboratory Tests in Elective Surgical Patients. The Internet Journal of Surgery, 30, 1-6.

[9] Chung, F., Yuan, H., Yin, L., Vairavanathan, S. and Wong, D. (2009) Elimination of Preoperative Testing in Ambulatory Surgery. Anesthesia \& Analgesia, 108, 467-475. https://doi.org/10.1213/ane.0b013e318176bc19

[10] National Collaborating Centre for Acute Care. NICE Guideline for Preoperative Investigations in Patients Undergoing Elective Surgery. Published June 2003.

[11] Kaplan, E.B., Sheiner, L.B., Boeckmann, A.J., Roizen, M.F., Beal, S.L., Cohen, S.N., et al. (1985) The Usefulness of Preoperative Laboratory Screening. The Journal of the American Medical Association, 253, 3576-3581.

https://doi.org/10.1001/jama.1985.03350480084025 